\title{
A Prediction Market System for Aggregating Dispersed Tacit Knowledge into a Continuous Forecasted Demand Distribution
}

\author{
Hajime Mizuyama and Eisuke Kamada \\ Dept. of Mechanical Engineering and Science, Kyoto University, \\ Kyoto, 606-8501, Japan \\ Telephone: +81-75-753-5237, Fax: +81-75-753-5239 \\ E-mail: \{mizuyama@mbox.kudpc.kyoto-u.ac.jp,e.kamada@gmail.com\} \\ WWW home page: http://www.users.kudpc.kyoto-u.ac.jp/ j54854/
}

\begin{abstract}
.
This research proposes a novel demand forecasting method which will work effectively even in such circumstances where extrapolate-able demand patterns are hardly available. The method uses the market mechanism to aggregate tacit knowledge of the firm's sales people on the future demand of a product into a continuous forecasted demand distribution. In order to make it work, the paper introduces a new type of prediction security and an original market maker algorithm suitable for the security, and furnishes them into an intra-firm prediction market system. As a result, sufficient liquidity is secured for the market even when the number of the traders is small, and the market maker can output at any time an aggregated demand forecast of the traders as a continuous distribution. An agent simulation model, where each trader has the log-utility function, is also developed to show how the method works, how quickly the output distribution converges, etc.
\end{abstract}

Keywords.

Demand forecasting, prediction markets, prediction market system, market maker, tacit knowledge.

\section{Introduction}

Most operations in a firm must be started before the actual demand for them has been known and fixed. Thus, the level of operation effectiveness a firm can reach depends inherently on how accurate it can forecast the future demand for its products. 
However, in the recent market environment characterized by product diversification and shortened product life cycles, accurate demand forecasting has become quite difficult. For example, now it is quite often that the future demand for a new product, whose historical sales data are not available, must be estimated. Further, even when predicting the demand for an existing product with its historical sales data, any patterns or trends found in the data rarely last long enough to be used to tell the future. Under such circumstances, the effectiveness of conventional forecasting methods, which extract certain structural patterns or trends in some past realized values of the demand (and maybe other related variables as well) and extrapolate them into the future, is obviously limited.

Thus, the authors have been developing a completely different framework for demand forecasting, which aggregates dispersed tacit knowledge owned by different people on the future demand into a forecast through the market mechanism. Laboratory experiments in experimental economics have confirmed that the market mechanism can aggregate information [1]. Further, Forsythe et al. [2] established a market on IEM (lowa Electronic Markets) of a virtual security whose payoff is tied to the vote share of a corresponding candidate in an election, and found that the price of the security usually gives a better forecast of the actual vote share than polls. Although it is not easy to establish a real-money market like IEM, Pennock et al. [3] and Servan-Schreiber et al. [4] confirmed that a play-money-based virtual market can also function as a forecasting machine and output a forecast as good as the one given by a real-money-based prediction market.

However, if a firm uses an open-structure prediction market composed of anonymous people for demand forecasting, a problem will arise that the result will also become open to public. Further, what a prediction market can do is not to create new information from nothing, but to aggregate existing dispersed information and reflect it into the forecast. Hence, the market should be composed of potentially knowledgeable people on the demand. Accordingly, what is suitable and effective for the purpose is rather a closed-structure prediction market composed, for example, of the firm's sales people, who should attain tacit knowledge on the future demand through their daily sales activities [1]. As far as the authors' knowledge, it is only Chen and Plott [5] that studies an intra-firm closed-structure prediction market applied to demand forecasting. In their work, the sales volume of a product is first divided into several mutually exclusive and collectively exhaustive intervals, for example, $[0,100],[101,200],[201,300], \ldots$, and a prediction security is issued to each of the predetermined intervals. Each unit of the fixed-interval prediction security pays off a unit amount of money if and only if the actual sales volume falls in the corresponding interval. Their market is composed of about 20 to 30 people, is run for about 1 week through simple double auction on computers, and yields a point estimate of the sales volume which is superior to the official forecast of the firm.

Two major problems should be pointed out to the above approach. One is that it is usually insufficient to obtain only a point estimate of the demand quantity. More important should be a distribution of the quantity, since, in practice, not only the mean but also the dispersion is critical input information to the following planning functions. With minor modifications so that the unitary price of the security can be deemed as the probability for the sales volume to fall in the corresponding interval, the above approach will become able to output a forecasted demand distribution as a 
histogram. However, the fixed width of each interval limits the accuracy and precision of the distribution from being improved along time through the dynamic nature of the market-based prediction. The other is that it is difficult to ensure sufficient liquidity for the prediction securities in a intra-firm market which is usually composed of only a small number of traders. A prediction market aggregates information through transactions of prediction securities, and hence will not function properly without sufficient liquidity. Chen and Plott [5] reported that arbitrage opportunities, which must soon disappear if the liquidity is sufficient, remained until the end in some market sessions. This represents that a market maker algorithm was necessary to supplement liquidity. For example, Hanson [6] and Pennock [7] each proposed a market maker algorithm for a prediction market. However, since prediction security design and market maker algorithm are not independent, it is important to deal with these issues together according to the characteristics of the forecasting problem.

\section{Intra-Firm Prediction Market System for Demand Forecasting}

\subsection{Variable Interval Prediction Security}

This section proposes an original prediction market system for obtaining not only a point estimate but also a continuous forecasted distribution of the demand quantity $x$ of a certain product in a predetermined period of time. In order to resolve the abovementioned limitation of the fixed interval security, the proposed system newly introduces the variable interval security. As is the case of the fixed interval security, each unit of the variable interval security pays off a unit amount of money if and only if the realized value of the demand quantity $x$ falls in the corresponding interval $[a, b]$. What is different is that not only the amount $v$ but also the interval $[a, b]$ of the security can be arbitrary specified by the trader when buying the security. Buying some additional security and selling some of the owned security are allowed at any time but, in order to simplify the cognitive load of the trader, only by changing the parameters $(a, b, v)$ of her/his position of the prediction security. Any such transactions are conducted at any time without liquidity problem with the computerized market maker to be described next.

\subsection{Market Maker Algorithm}

The market maker to be proposed here is furnished with a price density distribution $g(x)$, and offers a price to each unit of the variable interval prediction security with interval $[a, b]$ based on the distribution as:

$$
p(a, b)=\int_{a}^{b} g(x) d x
$$

Thus, when a trader changes her/his position of the prediction security from $(a, b, v)$ to $\left(a^{\prime}, b^{\prime}, v^{\prime}\right)$, the market maker will charge $p\left(a^{\prime}, b^{\prime}\right) v^{\prime}-p(a, b) v$. Since the realized value of $x$ must be contained in the interval $[0, \infty]$ and hence a unit of the security 
with this interval returns a unit amount of money without a risk, the following equation is satisfied:

$$
\int_{0}^{\infty} g(x) d x=1
$$

Further, any interval should not be given a negative price. Thus, the price density function satisfies the requirements of a probability density function. For the sake of simplicity, we will use a normal distribution $N\left(\mu_{g}, \sigma_{g}^{2}\right)$ for the function $g(x)$ in the following discussion.

The proposed system uses this function $g(x)$ also as the signal that feeds back each trader the information of the others and intermediates the micro-macro loop of learning for the traders to finally reach an agreed forecast. Therefore, the equilibrium of this market should satisfy not only that the transactions of the prediction security and the form of the price density function become stable, but also the following two conditions:

- When the subjective forecasted demand distributions are different among the traders, the price density function should provide each trader some information on the subjective distributions of the others.

- When the subjective forecasted distributions of the traders have become identical through the micro-macro loop interactions, the agreed forecasted distribution can be captured by the price density function.

The proposed market maker realizes these conditions by updating the price density $g(x)$ according to the transactions of the prediction security as follows:

Step 0: Initialize the parameters $\left(\mu_{g}, \sigma_{g}^{2}\right)$ of the price density function $g(x)$ so that they should represent the prior distribution of the demand quantity.

Step 1: Show each trader the current price density $g(x)$. When a trader changes or confirms her/his position of the prediction security to $(a, b, v)$, go to Step 2 .

Step 2: Update the parameters from $\left(\mu_{g}, \sigma_{g}^{2}\right)$ to $\left(\mu_{g}^{\prime}, \sigma_{g}^{\prime 2}\right)$ by the below equations:

$$
\begin{aligned}
& \mu_{g}^{\prime}=\mu_{g}+\alpha\left(m-\mu_{g}\right) \\
& \sigma_{g}^{\prime 2}=(1-\alpha)\left[\sigma_{g}^{2}+\left(\mu_{g}^{\prime}-\mu_{g}\right)^{2}\right]+\alpha\left[(r / 3)^{2}+\left(m-\mu_{g}\right)^{2}\right]
\end{aligned}
$$

where $m=(a+b) / 2, r=(b-a)$ and $\alpha=p(a, b) v / w_{0}$ (where $w_{0}$ is a given constant). Then, go back to Step 1. 


\section{Multi-Agent Simulation of Demand Forecasting System}

\subsection{Assumptions on Market and Traders}

This section studies the basic function of the proposed demand forecasting system through a simple multi-agent simulation. In the simulation, the following assumptions are made on the traders and the regulations of the market:

- There are 10 trader agents participated in the simulation market. Transactions of the prediction security are made one trader at a time after another with the market maker. The trader agent who conducts the transaction at each turn is selected through random sampling from the whole trader agents.

- Each trader agent $k$ has her/his own subjective forecasted distribution of the demand quantity $x$ based on her/his tacit knowledge. This subjective distribution $f_{k}(x)$ can be approximated by a normal distribution $N\left(\mu_{f, k}, \sigma_{f, k}^{2}\right)$.

- At the beginning of a market session, each trader agent is given a certain amount of money $u_{0}$, which can be either the real money or virtual money. When using the virtual money in practice, an incentive can be given to each trader by relating the final amount at hand to her/his work performance evaluation.

- The amount of the money at hand, the position of the prediction security, and the current value of the whole asset that the trader $k$ owns are denoted by $u_{k},\left(a_{k}, b_{k}\right.$, $v_{k}$ ) and $w_{k}$, respectively. Then, the whole asset at hand of each trader $k$ at the beginning is $w_{k}=u_{k}=u_{0}$, and the below equation holds at any time:

$$
w_{k}=p\left(a_{k}, b_{k}\right) v_{k}+u_{k}
$$

- Every trader agent $k$ has the $\log$ utility function of the money.

- Any short position of the money is not allowed for the traders at any time, so as to keep the effect of a single trader on the whole system controllable by the amount of the initial endowment $u_{0}$.

- Whereas short position of the prediction security is allowed as far as the money cannot become short. A short position of the prediction security in a certain interval is equivalent to the long position in the supplementary region.

In the following, the subscript $k$ representing the trader agent may be omitted, when this will not cause confuses.

When the asset value of a trader agent $k$ is $w \geq 0$, then the trader can take any position $(a, b, v)$ that satisfies equation (5) through a transaction of the prediction security. In this case, his/her subjective expected utility after the transaction is given by:

$$
E U=q(a, b) \log [w+\bar{p}(a, b) v]+\bar{q}(a, b) \log [w-p(a, b) v]
$$


where $q(a, b)$ represents the subjective probability that the value of $x$ falls in the interval $[a, b]$, and can be expressed with her/his subjective forecasted distribution $f(x)$ as:

$$
q(a, b)=\int_{i}^{b} f(x) d x
$$

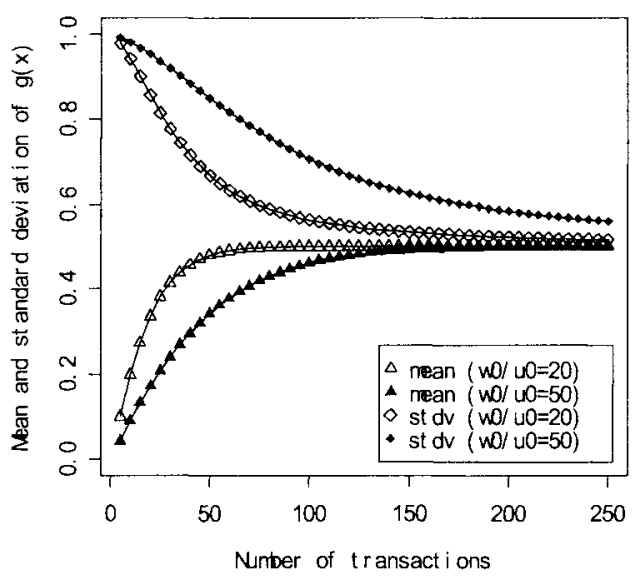

Fig. 1. How the price distribution changes along transactions in identical forecasts case

Further, $\bar{p}$ and $\bar{q}$ denote $(1-p)$ and $(1-q)$ respectively.

The trader $k$ will make a transaction so that this subjective expected utility in equation (6) should become maximized. At this moment, $w$ does not depend on the parameters $(a, b, v)$, and $p(a, b)$ and $q(a, b)$ are independent of $v$. Thus, the value of $v$ that maximizes equation (6) under a given $(a, b)$ can be easily derived as:

$$
v^{*}=\frac{q(a, b)-p(a, b)}{p(a, b) \bar{p}(a, b)} w
$$

When we substitute this $v^{*}$ for $v$ in the equation (6), then we have:

$$
E U^{*}=q(a, b) \log \left[\frac{q(a, b)}{p(a, b)}\right]+\bar{q}(a, b) \log \left[\frac{\bar{q}(a, b)}{\bar{p}(a, b)}\right]+\log (w)
$$

Hence, the trader will determine the interval $[a, b]$ of the prediction security to buy so as to maximize this $E U^{*}$. In the simulation, the parameters $(a, b, v)$ at each transaction are calculated as described. 


\subsection{Simulation Results}

First, we consider the case where the subjective forecasted distributions of the traders have become all identical. We set the prior price distribution as $g(x)=N(0,1)$, and investigate how this is being updated along transactions with different settings of the mean and the standard deviation of the identical subjective distribution $f(x)$, and the amount of the initial endowment $u_{0}$. Fig. 1 represents how the mean and the standard deviation of the price distribution change along transactions under $f(x)=N(0.5,0.5)$

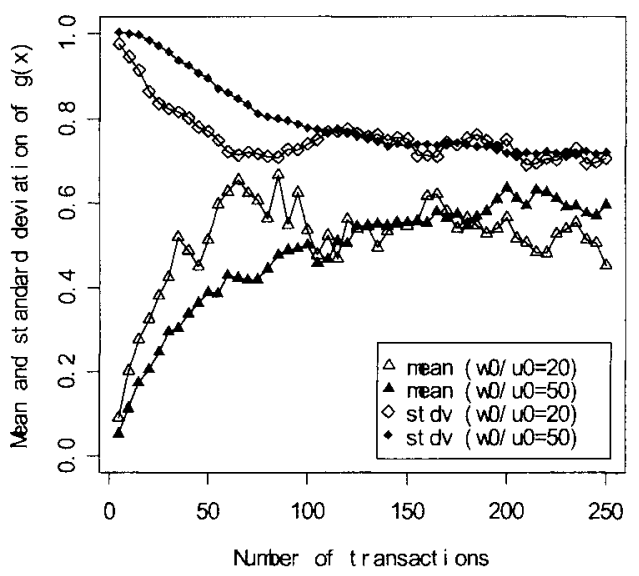

Fig. 2. How the price distribution changes along transactions in different forecasts case

and $w_{0} / u_{0}=20$ and 50. As seen in this chart, it is confirmed in any simulation experiments in this case that the price distribution will be finally converged upon the given identical subjective forecasted demand distribution and that the larger the amount of $u_{0}$ the quicker the converging process.

Next, we deal with the case where the subjective forecasted distributions are different among traders. In this case, we use the same prior price distribution $g(x)=$ $N(0,1)$, and set the parameters $\mu_{f k}$ and $\sigma_{f k}$ of each subjective distribution $f_{k}(x)$ by sampling from a uniform distributions $[0,1]$ and $[1 / 3,1 / 2]$ respectively. Fig. 2 represents an example of the results of similar simulation experiments. As seen in this chart, it is found that the price distribution tends to be converged upon a certain form even though the traders have different subjective forecasts. However, the convergence is not complete and some oscillation remains both in the mean and the standard deviation. A large amount of $u_{0}$ quickens the converging process but also enlarges the magnitude of the remained oscillation.

It is also confirmed that the values upon which the mean and the standard deviation of the price distribution converges are strongly correlated with the mean and the standard deviation of the compound distribution of $f_{1}(x), f_{2}(x), \ldots, f_{10}(x)$. This 
means that the converged distribution is capable of providing each trader some information on the subjective distributions of the others.

\section{Conclusions}

This paper presented a fundamental design of an intra-firm prediction market system for aggregating tacit knowledge of the sales people into a continuous forecasted demand distribution, and studied its basic function through a simple multi-agent simulation. It is observed that the output distribution converges upon a desirable form and that the larger the amount of the initial endowment the quicker the converging process. The simulation is run on the assumption that each trader has the $\log$ utility function, her/his subjective demand forecast can be represented by an unknown normal distribution, and she/he can precisely determine the optimum asset position when conducting a transaction. It is also important to investigate through the simulation how the system will work when these assumptions are relaxed and further when a learning capability is provided to each trader agent. Following such thorough simulation-based study, laboratory experiments and field experiments will be necessary so as to proceed to the stage of real-life application.

\section{References}

1. C.R. Plott, Markets as Information Gathering Tools, Southern Economic Journal, 67, 1-15 $(2000)$

2. R. Forsythe, T.A. Rietz and T.W. Ross, Wishes, Expectations and Actions: A Survey on Price Formation in Election Stock Markets, Journal of Economic Behavior \& Organization, 39, 83-110 (1999).

3. D.M. Pennock, S. Lawrence, C.L. Giles and F.A. Nielsen, The Real Power of Artificial Markets, Science, 291, 987-988 (2001).

4. E. Servan-Schreiber, J. Wolfers, D.M. Pennock and B. Galebach, Prediction Markets: Does Money Matter?, Electronic Markets, 14, 243-251 (2004).

5. K. Chen and C.R. Plott, Information Aggregation Mechanisms: Concept, Design and Implementation for a Sales Forecasting Problem, California Institute of Technology Social Science Working Paper, \#1131, (2002).

6. R. Hanson, Combinatorial Information Market Design, Information Systems Frontiers, 5 , 107-119 (2003).

7. D.M. Pennock, A Dynamic Pari-Mutuel Market for Hedging, Wagering, and Information Aggregation, Proceedings of the 5th ACM conference on Electric Commerce, 170-179 (2004). 